\title{
Assessment of heavy metals concentrations in water, plankton and fish of Lake Manzala, Egypt
}

\author{
Mohamed H. Bahnasawy, Abdel Aziz A. Khidr, Nadia A. Dheina \\ Zoology Department, Damietta Faculty of Science, Damietta, Egypt
}

\begin{abstract}
The levels of some heavy metals $(\mathrm{Cu}, \mathrm{Zn}, \mathrm{Cd}, \mathrm{Pb})$ were determined in water, plankton and fish (Liza aurata) collected from five sites in Lake Manzala. Metals in water and fish exhibited a significant seasonal and regional variations in which all metals attained their maximum values during summer, while the lowest level was reported during winter. The accumulation of different metals in water, plankton and fish tissues followed the order $\mathrm{Zn}>\mathrm{Cu}>\mathrm{Pb}>\mathrm{Cd}$. The mean concentrations of the tested metals in water were: $\mathrm{Cu}(0.055), \mathrm{Zn}(0.311), \mathrm{Cd}$ $(0.020)$ and $\mathrm{Pb}(0.022) \mathrm{mg} / \mathrm{l}$. Cd level in water was found to be higher than the permissible limit recommended for drinking water. Metals in plankton were much higher than those in water and fish. Gills of the examined fish contained the highest concentrations of all the measured metals, while muscles retained the lowest levels. In spite of the contamination of Lake Manzala by such heavy metals, the levels of these metals in the edible fish muscle did not exceed the recommended permissible limits and thus are considered safe for human consumption.
\end{abstract}

Key words: Lake Manzala, heavy metals, water, plankton, fish.

\section{INTRODUCTION}

In aquatic systems, heavy metals have received considerable attention due to their toxicity and accumulation in biota (Mason, 1991). Metals generally enter the aquatic environment through atmospheric deposition, erosion of geological matrix or due to anthropogenic activities caused by industrial effluents, domestic sewage and mining wastes (Tarvainen et al.1997; Stephen et al., 2000). Some of these metals are toxic to living organisms even at quite low concentrations, whereas others are biologically essential as natural constituents of aquatic ecosystem and only become toxic at very high concentrations.

Heavy metals may affect organisms directly by accumulating in the body or indirectly by transferring to the next trophic level of the food chain. Being non-biodegradable like many organic pollutants, they can be concentrated along the food chain, producing their toxic effects at points often far away from the source of pollution (Fernandez et al., 2000). Accumulation of heavy metals in the food web can occur either by accumulation from the surrounding medium such as water or sediment, or by bioaccumulation from the food source (Tulonen et al., 2006). Aquatic organisms have been widely used in biological monitoring and assessment of safe environmental levels of heavy metals. 
Lake Manzala is one of the large lakes in northern region of Egypt (about 52611 hectares surface area) and the most productive for fisheries. The lake receives heavy loads of organic and inorganic pollutants via several agricultural drains (Badawy et al., 1995). Due to the toxicity of heavy metals, accurate information about their concentration in aquatic ecosystem is needed (Janssen et al., 2000). Therefore, the objective of this study was to evaluate the pollution level of Lake Manzala via determining the accumulation of $\mathrm{Cu}, \mathrm{Zn}, \mathrm{Cd}$ and $\mathrm{Pb}$ in water, plankton and some tissues of Liza aurata.

\section{MATERIALS AND METHODS}

Lake Manzala is bounded by the Mediterranean Sea to the north, the Suez canal to the east and Damietta branch of Nile to the west (Fig.1).

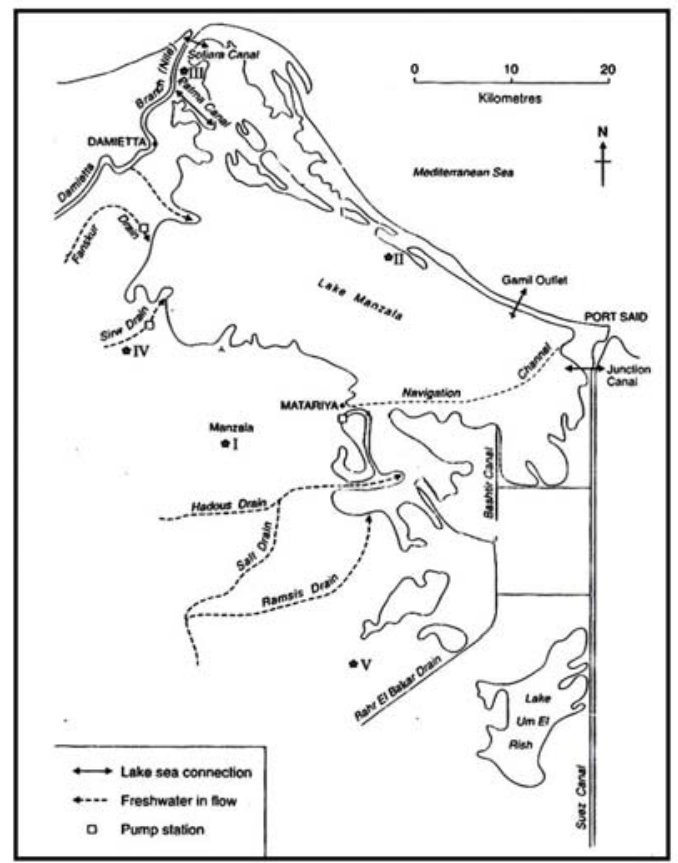

Figure (1): Location of sampling sites $\left(^{*}\right)$ in Lake Manzala; El-Manzala (I), El-Diba (II), ElRatama (III), El-Sirw (IV) and Bahr El-Bakar (V)

The lake has gradually changed from a brackish environment to eutrophic freshwater basin due to the increased amounts of agricultural drainage water and sewage discharge into it via seven major drains (Abdel-Baky et al., 1998). Water, plankton and fish (Liza aurata, mugilidae) samples were collected from five different locations in the Lake (Fig.1) during four seasons from winter 2001 to autumn 2002. The locations were chosen to represent different levels of pollution. Water samples were collected monthly from $50 \mathrm{~cm}$ depth in two liters 
polyethylene bottle acidified with nitric acid and kept for analysis. Plankton (zoo and phytoplankton) samples were collected with a plankton net of $55 \mu \mathrm{m}$ mesh size, through vertical hauls from the upper $10 \mathrm{~cm}$ layer. Filtered plankton samples were acidified with $\mathrm{HCl}$ and kept for analysis. Parts of gills, skin and dorsal muscle were taken from each fish, weighed, put in small Erlenmeyer flasks, dried in an oven at $105^{\circ} \mathrm{C}$ for about 24 hours and digested by conc. Nitric acid and perchloric acid on a hotplate until the solution became clear. $\mathrm{Cu}, \mathrm{Zn}, \mathrm{Cd}$ and $\mathrm{Pb}$ concentrations in water were determined by extraction method (APHA, 1998) using atomic absorption spectrophotometer. Plankton and fish samples were prepared for heavy metals analysis according to the method described by Kalay et al.(1999). Two-way ANOVA was employed to find the significant differences of heavy metals concentration in water, plankton and fish organs with regard to sites and seasons (Bailey, 1982).

\section{RESULTS AND DISCUSSION}

The mean concentrations of $\mathrm{Cu}, \mathrm{Zn}, \mathrm{Cd}, \mathrm{Pb}$ in water samples collected from Lake Manzala are shown in Table 1. The mean concentrations of the tested metals in water were found in the following order: $\mathrm{Cd}(0.020)<\mathrm{Pb}(0.022)<$ $\mathrm{Cu}(0.055)<\mathrm{Zn}(0.311) \mathrm{mg} / \mathrm{l}$. This order of occurrence agrees with the previous studies performed on Lake Manzala (Abdel-Baky et al., 1998; Ibrahim et al., 1999). All the metals attained their maximum values at site $\mathrm{V}$ which receives huge quantities of sewage and industrial wastes, beside agricultural drainage water via Bahr Al-Bakar drain. Badaway and Wahaab (1997) reported that water in Bahr Al-Bakar region is not suitable for human use. It was found that this site is rich in organic carbon (Dheina, 2007) and some authors found a correlation between the concentration of heavy metals in water and the abundance of organic matter (Radwan et al.1990a; Abdel-Baky et al., 1998). Site II appeared to be the least polluted region of the lake as it contained the lowest levels of the investigated metals. Since it did not receive much of agricultural, industrial and sewage drains. The levels of metals exhibited seasonal fluctuations, where $\mathrm{Cu}$, $\mathrm{Cd}, \mathrm{Pb}$ showed significant differences between seasons. Their highest levels were found during summer, while their lowest values occurred during winter. These seasonal variations may be due to the fluctuation of the amount of agricultural drainage water, sewage effluents and industrial wastes discharged into the lake (Zyada, 1995). Ali and Abdel-Satar (2005) attributed the increase of metals concentration in water during hot seasons (spring and summer) to the release of heavy metals from sediment to the overlying water under the effect of both high temperature and fermentation process due to decomposition of organic matter. The seasonal variations of metals in water were reported by different authors in different water dodies: El-Safy and Al-Ghannam (1996), Abdel-Baky et al.(1998), Ibrahim et al. (1999) in Lake Manzala, Hamed(1998) in River Nile. Compared with the previous studies of Lake Manzala, Abdel-Hamid and El-Zareef (1996) found lower values of $\mathrm{Cu}$ (0.01-0.02)mg/l, El-Safy and 
Al-Ghannam (1996) obtained lower Cd but higher Pb, Abdel-Baky et al.(1998) recorded higher values of $\mathrm{Cu}$ (0.08), $\mathrm{Zn}$ (7.94), $\mathrm{Cd}$ (0.11), $\mathrm{Pb}(0.64) \mathrm{mg} / \mathrm{l}$, Ibrahim et al.(1999) reported higher value of $\mathrm{pb}(0.09)$ but lower levels of $\mathrm{Cu}$ (0.03), Zn (0.23) and Cd (0.005) mg/l. Compared with other lakes, $\mathrm{Cu}, \mathrm{Zn}, \mathrm{Cd}$, $\mathrm{Pb}$ in Lake Manzala are higher than those of Piaseczno Lake (Poland) (0.015, 0.058, 0.001, $0.018 \mathrm{mg} / 1$ respectively) (Radwan et al,1990a), Lapland Lake (Finland) had higher Zn (1.84 mg/l) (Mannio et al.,1995), Dominic Lake had higher $\mathrm{Cu}$ (3.93mg/l) (Szymanowska et al.,1999). Higher concentrations of Cd (0.11) and $\mathrm{Pb}(0.086) \mathrm{mg} / \mathrm{l}$ were found in Beysehir Lake, Turkey (Altindag and Yigit,2005). Uluabat Lake (Turkey) contained higher $\mathrm{Cu}(0.14), \mathrm{Cd}(0.04), \mathrm{Pb}$ (0.03) $\mathrm{mg} / \mathrm{l}$ (Elmaci et al.,2007).

According to USEPA (1986) $\mathrm{Cu}, \mathrm{Zn}, \mathrm{Pb}$ levels in Lake Manzala were within the permissible limit recommended for drinking and irrigation purposes, while that of $\mathrm{Cd}$ was found higher than those recommended.

Studying of heavy metals concentrations in plankton is very important because plankton is often the main diet for many predators and may remarkably contribute to the transfer of heavy metals to higher trophic levels. The results (Table2) indicate that $\mathrm{Cu}, \mathrm{Zn}, \mathrm{Cd}, \mathrm{Pb}$ concentrations in plankton were much higher than those of water. This may be related to the large surface of plankton organisms (phyto + zooplankton) in relation to their mass unit, and their active metabolism leading to rapid adsorption of various pollutants (Ravera, 2001). The latter author added that some algal species protect themselves by trapping and accumulating pollutants (e.g metals) in their polysaccharides wall. The order of abundance of metals in plankton was $\mathrm{Zn}>\mathrm{Cu}>\mathrm{Pb}>\mathrm{Cd}$. This corresponds to the same order of abundance of these metals in water, which support the hypothesis that water is an important source of plankton contamination. Elmaci et al.(2007) reported that the quantity of heavy metals in plankton depends on their concentration in water and partially on sediment. All the metals in plankton attained their maximum values at site $\mathrm{V}$, where its water had the highest concentrations of these metals. The accumulation of heavy metals in plankton has been reported to depend upon several factors, such as the productivity of water body, the physico-chemical properties of the water, quantitative and qualitative species composition of zoo and phytoplankton, capacity of heavy metals absorbance and season (Radwan et al., 1990b; Kerrison et al., 1998; Elmaci et al., 2007). There were no significant differences in metals in plankton between sites and seasons. Compared with other studies, small plankton and macro zooplankton from American lakes accumulated lower levels of $\mathrm{Cu}, \mathrm{Zn}$, $\mathrm{Cd}, \mathrm{Pb}$ (Chen et al., 2000). Plankton from lakes in southern Finland showed higher level of $\mathrm{Cu}$ but lower levels of $\mathrm{Cd}, \mathrm{Zn}, \mathrm{Pb}$ (Tulonen et al., 2006). Elmaci et al. (2007) recorded enormously higher concentrations of $\mathrm{Cu}$ (6820.0), $\mathrm{Zn}$ (20290.0), Cd (1450.0), Pb (580.0) $\mu \mathrm{g} / \mathrm{g}$ dry weight.

Heavy metals concentration in muscle, skin and gills of Liza aurata are shown in Tables (3-6). There were significant differences between sites, seasons 
and fish organs. The highest concentrations of $\mathrm{Cu}, \mathrm{Zn}, \mathrm{Cd}, \mathrm{Pb}$ were found in tissues of fish from site $\mathrm{V}$, where its water contained the highest levels of the measured metals. This agrees with Shakweer (1998) who concluded that the level of bioaccumulation of trace metals in various organs of fish reflects the degree of water pollution in aquatic environment in which such fish are living. Ravera (2001) reported that if an environment receives foreign pollutants the organism living in it will take up the pollutants from the water or/and food, and concentrate it in its body. The order of detection of metals in the fish organs was as follow: gills $>$ skin $>$ muscles. Gills accumulated the highest level of $\mathrm{Zn}$ (62.018-99.80), $\mathrm{Cu}$ (11.88-15.48), $\mathrm{Pb}(6.9-10.26) \mathrm{Cd}$ (2.93-5.19), $\mu \mathrm{g} / \mathrm{g}$ dry weight. The high content of metals in gill tissues can be attributed to the fact that fish gills play a distinct role in metal uptake from the environment. Due to its respiratory function, gills are in direct contact with the contaminated water and have the thinnest epithelium of all organs (Kotze et al., 1999). This result agrees with many authors who reported that gills have a high tendency to accumulate heavy metals (Unlu et al., 1996; Kotze et al., 1999; Wong et al., 2001; Coetzee et al., 2002; Altindag and Yigit, 2005). Compared with other studies, gills in the present study showed higher concentrations of $\mathrm{Cu}, \mathrm{Zn}, \mathrm{Cd}, \mathrm{Pb}$ than those reported in Mugil cephalus from northeast Mediterranean Sea (Canli and Atli, 2003). Following the gills, the skin accumulated lesser concentrations of the metals. The skin tissue together with the gill tissues are characterized by a mucus layer on the outer surface. This can indicate them as excretion routes involving the slaughing off mucus from their surface (Varanci and Markey, 1978, Yilmaz 2003). Skin of Mugil cephalus from Iskenderun Bay (Turkey) accumulated higher levels of $\mathrm{Pb}$ and $\mathrm{Zn}$ (Yilmaz, 2005). Muscles retained the lowest concentrations of the measured metals. This finding confirms the observations of many authors who showed that fish muscles have low tendency to accumulate heavy metals to which they are exposed (Blasco et al., 1998, Canli et al., 1998, Ibrahim et al.,1999, Canli and Atli, 2003, Karaded et al.,2004, Yilmaz, 2005). In light of the recommended permissible limits of heavy metals in fish tissue for human consumption according to the National Health Medical Research Council (NHMRC) (cited from Ibrahim et al.,1999b), it can be declared that the muscles of Liza aurata, in the present study, are considered safe for human consumption. Metals concentrations in fish organs exhibited seasonal variations in which all the detected metals attained their highest levels during summer, while their lowest values were found during winter. These seasonal variations can be attributed to the increase or decrease of drainage water discharged into the lake (Abdel-Baky et al., 1998). Compared with other studies, Liza aurata from the middle eastern Coast of Tunisia accumulated in their muscle higher levels of $\mathrm{Cu}$ (4.78), $\mathrm{Zn} \mathrm{(45.0)} \mathrm{but} \mathrm{lower} \mathrm{level} \mathrm{of} \mathrm{Cd}(0.07)$ $\mu \mathrm{g} / \mathrm{g}$ dry weight (Hamza-Chaffai et al.,1996). Enormously higher concentrations of $\mathrm{Cu}$ (23.16) and $\mathrm{Zn}(27.26) \mu \mathrm{g} / \mathrm{g}$ wet weight were found in muscle of Liza abu from Tigris River (Turkey) (Unlu et al., 1996). Blasco et al.(1998) measured a 
remarkably high concentration of $\mathrm{Cu}$ and $\mathrm{Zn}$ in muscle of Liza aurata from Cadiz Bay (Spain). Higher levels of $\mathrm{Cu}, \mathrm{Zn}, \mathrm{Cd}, \mathrm{Pb}$ were detected also in muscles of Liza ramada from Lake Manzala and from Damietta Nile Estuary (Ibrahim et al., 1999a\&b). Higher concentrations of $\mathrm{Zn} \mathrm{(37.39),} \mathrm{Pb} \mathrm{(5.32),} \mathrm{Cu}$ (4.41) but lower Cd (0.66) $\mu \mathrm{g} / \mathrm{g}$ dry weight were recorded in muscle of Mugil cephalus from the north east Mediterranean Sea (Canli and Atli, 2003).

\section{CONCLUSION}

Results of the present study clearly demonstrate that Lake Manzala is highly contaminated with $\mathrm{Cu}, \mathrm{Zn}, \mathrm{Cd}$ and $\mathrm{Pb}$ due to the continuous discharge of different pollutants into it. Great efforts and co-operation between different authorities are needed to protect the lake from pollution and reduce environmental risk. This can be achieved by treatment of the agricultural, industrial and sewage discharges. Regular evaluation of pollutants in the lake is also very important.

\section{REFERENCES}

Abdel-Baky, T. E.; Hagras, A. E.; Hassan, S. H. and Zyadah, M. A (1998). Environmental impact assessment of pollution in Lake Manzalah, 1Distribution of some heavy metals in water and sediment. J. Egypt. Ger. Soc. Zool., 26 (B): 25-38.

Abdelhamid, A. M. and El-Zareef, A. A. (1996). Further studies of the pollution status on the southern region of El-Manzalah Lake. Proc. Conf. Food Borne contamination and Egyptian's Health, Mansoura, Nov., 26-27, pp: 141-150.

Ali, M.H. and Abdel-Satar, A.M. (2005). Studies of some heavy metals in water, sediment, fish and fish diets in some fish farms in El-Fayoum province, Egypt. J. Aquat. Res., 31(2):261-273.

Altindag, A. and Yigit, S. (2005). Assessment of heavy metal concentrations in the food web of lake Beysehir, Turkey. Chemosphere, 60: 552-556.

APHA (1998): Standard methods for the examination of water and wastewater. $20^{\text {th }}$ Edition. American Public Health Association, Bultimore, USA.

Badawy, M. I.; Wahaab, R. A. and Abou Waly, H. F. (1995). Petroleum and chlorinated hydrocarbons in water from Lake Manzala and associated canals. Bull. Environ. Contam. Toxicol. 55: 258-263. 
Badawy, M. I. and Wahaab, R. A. (1997). Environmental impact of some chemical pollutants on Lake Manzala. International Journal of Environmental Health Research. 7: 161-170.

Bailey, N. (1982). Statistical methods in biology. $2^{\text {nd }}$ Ed. (Biological Science Texts), pp: 216.

Blasco, J.; Rubio, J. A.; Forja, J.; Gomezparra, A. and Establier, R. (1998). Heavy metals in some fishes of the Mugilidae family from salt ponds of Cadiz Bay, Spain. Ecotoxicology and Environmental Restoration, 1 (2): 71-77.

Canli, M.; Ay, Ö. and Kalay, M. (1998). Levels of heavy metals (Cd, $\mathrm{Pb}, \mathrm{Cu}, \mathrm{Cr}$ and $\mathrm{Ni}$ ) in tissues of Cyprinus carpio, Barbus capito and Chondrostoma regium from the Seyhan River, Turkey. Turkish, J. Zoology, 22: 149-157.

Canli, M. and Atli, G. (2003). The relationships between heavy metal $(\mathrm{Cd}, \mathrm{Cr}$, $\mathrm{Cu}, \mathrm{Fe}, \mathrm{Pb}$, and $\mathrm{Zn}$ ) levels and the size of six Mediterranean fish species. Environmental Pollution, 121: 129-136.

Chen,C.Y.; Stemberger, R. S.; Klaue, B.; Blum, J. D.; Pickhardt, P. C. and Folt, C. L. (2000). Accumulation of heavy metals in food web components across a gradient of lakes. Limnol.Oceanogr.,45(7): 1525-1536.

Coetzee, L.; du Preez, H. H. and van Vuren, J. H. J. (2002). Metal concentrations in Clarias gariepinus and Labeo umbratus from the Olifant and Klein Olifants River, Mpumalanga, South Africa: Zinc, copper, manganese, lead, chromium, nickel, aluminium and iron. Water SA, 28 (4): 433-448.

Dheina, N.A. (2007). Biological and ecological studies on some species of family Mugilidae from different habitats in relation to their parasitic fauna. Ph. D. Thesis. Damietta Faculty of Science, Mansoura University, Egypt.

Elmaci, A.; Teksoy, A.; OlcayTopac, F.; Ozengin, N.; Kurtoglu, S. and Savas Baskaya, H. (2007).Assessment of heavy metals in lake Uluabat, Turkey. Afr. J. Biotech., 6 (19): 2236-2244.

El-Safy, M. K. and Al-Ghannam, M. L. (1996). Studies on some heavy metal pollutants in fish of El-Lake Manzala. Proc. Conf. Food Borne Contamination and Egyptians Health, Mansoura, Nov. 26-27, pp.151-180. 
Fernandez, M.; Cuesta, S.; Jimene, O.; Garcia, M. A. Hernandez, L.M.; Marina, M. L. and Gonzalez, M. J.(2000). Organochlorine and heavy metal residues in the water, sediment system of the southeast regional park in Madrid, Spain. Chemosphere, 41:801-812.

Hamed, M. A. (1998). Distribution of trace metals in the River Nile ecosystem Damietta branch between Mansoura city and Damietta province. J. Egypt. Ger. Soc. Zool., 27 (A): 399-415.

Hamza-Chaffai, A.; Romeo, M. and El-Abed, A. (1996). Heavy metals in different fishes from the Middle Eastern coast of Tunisia. Bull. Environ. Contam. Toxico., 56: 766-773.

Ibrahim, A. M.; Bahnasawy, M. H.; Mansy, S. E. and El-Fayomy, R. I. (1999a). Heavy metal accumulation in water, Sediment and some fishes in Lake Manzala, Egypt. J. Egypt. Ger. Soc. Zool., 29 (B): 43-58.

Ibrahim, A. M.; Bahnasawy, M. H.; Mansy S. E. and EL-Fayomy, R. I. (1999b). Distribution of heavy metals in the Damietta Nile Estuary ecosystem. Egypt. J. Aquat. Biol \& Fish., 3(4): 369-397.

Ibrahim, A. M.; Bahnasawy, M. H.; Mansy, S. E. and EL-Fayomy, R. I. (2000). On some heavy metal levels in water, sediment and marine organisms from the Mediterranean coast of Lake Manzala. Egypt. J. Aquat. Biol \& Fish., 4(4): 61-81.

Janssen, C.R., DeSchamphelaere, K., Heijerick, D., Muyssen, B., Lock, K., Bossuyt, B., Vangheluwe, M. and VanSprang, P.(2000). Uncertainties in the environmental risk assessment of metals. Hum.Ecol.Risk.Assess.,6: 1003-1018.

Kalay, M.; Ay, Ö. and canli, M. (1999). Heavy metal concentrations in fish tissues from the Northeast Mediterrenean Sea. Bull. Environ. Contam. Toxicol., 63: 673-681.

Karadede, H.; Oymak, S. A. and Ünlü, E. (2004). Heavy metals in mullet, Liza $a b u$, and Catfish, Silurus triostegus, from the Atatürk Dam Lake (Euphrates), Turkey. Environment Internationl, 30: 183-188.

Kerrison, P.H., Annonsi, D., Zerini, S., Ravera, O. and Moss. B. (1998). Effects of low concentrations of heavy metals on plankton community dynamics in a small, shallow, fertile lake. J. Plankton Res.,10:779-812. 
Kotze, P.; Du preez, H. H. and Van Vuren, J. H. (1999). Bioaccumulation of copper and zinc in Oreochromis mossambicus and Clarias gariepinus, from the Olifants River, Mpumalanga, South Africa. water S. A., 25 (1):99-110.

Mannio,J., Jarvien, O., Tuominen,R. and Verta, M. (1995). Survey of trace elements in lake waters of Finnish Lapland using the ICP-MS technique. Sci. Total Environ.,160-161: 433-439.

Mason, C. (1991). Biology of freshwater pollution. Harlow: Longman Scientific and Technical.

Radwan, S.; Kowalik, W. and Kornijow, R. (1990a). Accumulation of heavy metals in a Lake ecosystem. Sci. Total Environ., 96: 121-129.

Radwan, S.; Kowalik, W. and Kowalczyk, C. (1990b). Occurrence of heavy metals in water, phytoplankton and zooplankton of mesotrophic Lake in Estern Poland. Sci. Total Environ., 96: 115-120.

Ravera, O. (2001). Monitoring of the aquatic environment by species accumulator of pollutants: a review. J. imnol.,60: 63-78.

Shakweer, L. M. (1998). Concentration levels of some trace metals in Oreochromis niloticus at highly and less polluted areas of Mariut Lake. J. Egypt. Ger. Soc. Zool., 25(A): 125-144.

Stephen, C.; Jewett, A. and Sathy Naidu, A. (2000). Assessment of heavy metals in red king crabs following offshore placer gold mining. Mar. Pollut. Bull., 40(6): 478-490.

Szymanowska, A.; Samecka-Symerman, A. and Kampers, A. J. (1999). Heavy metals in three lakes in west Poland. Ecotoxicol. Environ. Safety, 43: 21-29.

Tarvainen, T.; Lahermo, P. and Mannio, J. (1997). Source of trace metals in streams and headwater lakes in Finland. Water, Air, Soil Pollut., 94: 1-32.

Tulonen,T.; Pihlstrom, M.; Arvola, L. and Rask, M.(2006). Concentrations of heavy metals in food web components of small, boreal lakes. Boreal Environ. Res., 11:185-194

U. S. E. P. A. (United States Environmental Protection Agency) (1986). Quality criteria for water. 1986. EPA 440 15-86-001. May 1986. 
Ünlü, E.; Akba, O.; Sevim, S. and Gümgüm, B. (1996). Heavy metal levels in mullet, Liza abu (Heckel, 1843) (Mugilidae) from the Tigris River, Turkey. Fresenius Envir. Bull., 5: 107-112.

Varanasi, U. and Markey, Y. D. (1978). Uptake and release of lead and cadmium in skin and mucus of coho salmon (Oncorhynchus kisutch). Comp. Biochem. Physiol. 60C: 187-191.

Wong, C.K.; Wong, P. P. K. and Chu, L. M. (2001). Heavy metal concentrations in marine fishes collected from fish culture sites in Hong Kong. Arch. Environ.Contam.Toxicol.40:60-69.

Yilmaz, A.B. (2003). Levels of heavy metals ( $\mathrm{Fe}, \mathrm{Cu}, \mathrm{Ni}, \mathrm{Cr}, \mathrm{Pb}$ and $\mathrm{Zn}$ ) in tissue of Mugil cephalus and Trachurus mediterraneus from Iskenderun Bay, Turkey. Environ. Res., 92: 277-281.

Yilmaz, A.B.(2005). Comparison of heavy metal levels of grey mullet (Mugil cephalus) and sea bream (Sparus aurata) caught in Iskenderun Bay (Turkey). Turk. J. Vet. Anim. Sci., 29:257-262. 
Table 1: Seasonal variations of heavy metals concentrations (mg/l) in water of Lake Manzala.

\begin{tabular}{|c|c|c|c|c|c|c|c|c|c|c|}
\hline \multirow{2}{*}{ Elements } & \multirow{2}{*}{ Site } & \multicolumn{5}{|c|}{ Seasons } & \multicolumn{4}{|c|}{ ANOVA } \\
\hline & & Winter & Spring & Summer & Autumn & Total & Factor & df & $F$ value & Sig. \\
\hline \multirow{6}{*}{$\mathrm{Cu}$} & I & $\begin{array}{c}0.038 \\
\pm \\
0.002\end{array}$ & $\begin{array}{c}0.051 \\
\pm \\
0.042\end{array}$ & $\begin{array}{c}0.061 \\
\pm \\
0.02\end{array}$ & $\begin{array}{c}0.040 \\
\pm \\
0\end{array}$ & $\begin{array}{c}0.048 \\
\pm \\
0.031\end{array}$ & & & & \\
\hline & II & $\begin{array}{c}0.009 \\
\pm \\
0 \\
\end{array}$ & $\begin{array}{c}0.028 \\
\pm \\
0.004 \\
\end{array}$ & $\begin{array}{c}0.031 \\
\pm \\
0.013 \\
\end{array}$ & $\begin{array}{c}0.016 \\
\pm \\
0.029 \\
\end{array}$ & $\begin{array}{c}0.021 \\
\pm \\
0.009 \\
\end{array}$ & Site & 4 & 16.096 & 0 \\
\hline & III & $\begin{array}{c}0.025 \\
\pm \\
0.003\end{array}$ & $\begin{array}{c}0.040 \\
\pm \\
0.004 \\
\end{array}$ & $\begin{array}{c}0.055 \\
\pm \\
0.009 \\
\end{array}$ & $\begin{array}{c}0.038 \\
\pm \\
0.007\end{array}$ & $\begin{array}{c}0.040 \\
\pm \\
0.004 \\
\end{array}$ & Season & 3 & 14.288 & 0 \\
\hline & IV & $\begin{array}{c}0.032 \\
\pm \\
0.002\end{array}$ & $\begin{array}{c}0.065 \\
\pm \\
0.002\end{array}$ & $\begin{array}{c}0.083 \\
\pm \\
0.004\end{array}$ & $\begin{array}{c}0.049 \\
\pm \\
0.004\end{array}$ & $\begin{array}{c}0.057 \\
\pm \\
0.004\end{array}$ & Site $\mathrm{x}$ Season & 12 & 13.479 & 0 \\
\hline & V & $\begin{array}{c}0.053 \\
\pm \\
0.009\end{array}$ & $\begin{array}{c}0.111 \\
\pm \\
0.002 \\
\end{array}$ & $\begin{array}{c}0.192 \\
\pm \\
0.002\end{array}$ & $\begin{array}{c}0.088 \\
\pm \\
0.02\end{array}$ & $\begin{array}{c}0.111 \\
\pm \\
0.007\end{array}$ & & & & \\
\hline & Total & $\begin{array}{c}0.031 \\
\pm \\
0.003\end{array}$ & $\begin{array}{c}0.059 \\
\pm \\
0.011\end{array}$ & $\begin{array}{c}0.084 \\
\pm \\
0.010 \\
\end{array}$ & $\begin{array}{c}0.046 \\
\pm \\
0.012\end{array}$ & $\begin{array}{c}0.055 \\
\pm \\
0.011 \\
\end{array}$ & & & & \\
\hline \multirow{6}{*}{$\mathrm{Zn}$} & I & $\begin{array}{c}0.177 \\
\pm \\
0.221\end{array}$ & $\begin{array}{c}0.370 \\
\pm \\
0.136\end{array}$ & $\begin{array}{c}0.472 \\
\pm \\
0.179\end{array}$ & $\begin{array}{c}0.246 \\
\pm \\
0.112\end{array}$ & $\begin{array}{c}0.316 \\
\pm \\
0.154\end{array}$ & & & & \\
\hline & II & $\begin{array}{c}0.139 \\
\pm \\
0\end{array}$ & $\begin{array}{c}0.281 \\
\pm \\
0.058\end{array}$ & $\begin{array}{c}0.301 \\
\pm \\
0.065\end{array}$ & $\begin{array}{c}0.198 \\
\pm \\
0.009\end{array}$ & $\begin{array}{c}0.230 \\
\pm \\
0.031\end{array}$ & Site & 4 & 2.421 & 0.064 \\
\hline & III & $\begin{array}{c}0.181 \\
\pm \\
0.013\end{array}$ & $\begin{array}{c}0.310 \\
\pm \\
0.161 \\
\end{array}$ & $\begin{array}{c}0.372 \\
\pm \\
0.013\end{array}$ & $\begin{array}{c}0.226 \\
\pm \\
0.114\end{array}$ & $\begin{array}{c}0.272 \\
\pm \\
0.058 \\
\end{array}$ & Season & 3 & 3.156 & 0.035 \\
\hline & IV & $\begin{array}{c}0.198 \\
\pm \\
0.047\end{array}$ & $\begin{array}{c}0.382 \\
\pm \\
0.036\end{array}$ & $\begin{array}{c}0.493 \\
\pm \\
0.147\end{array}$ & $\begin{array}{c}0.288 \\
\pm \\
0.042\end{array}$ & $\begin{array}{c}0.340 \\
\pm \\
0.257\end{array}$ & Site $\mathrm{x}$ Season & 12 & 2.726 & 0.009 \\
\hline & V & $\begin{array}{c}0.232 \\
\pm \\
0.226 \\
\end{array}$ & $\begin{array}{c}0.470 \\
\pm \\
0.087\end{array}$ & $\begin{array}{c}0.529 \\
\pm \\
0.183\end{array}$ & $\begin{array}{c}0.352 \\
\pm \\
0.031 \\
\end{array}$ & $\begin{array}{c}0.396 \\
\pm \\
0.143 \\
\end{array}$ & & & & \\
\hline & Total & $\begin{array}{c}0.185 \\
\pm \\
0.101\end{array}$ & $\begin{array}{c}0.363 \\
\pm \\
0.096 \\
\end{array}$ & $\begin{array}{c}0.433 \\
\pm \\
0.117 \\
\end{array}$ & $\begin{array}{c}0.262 \\
\pm \\
0.062\end{array}$ & $\begin{array}{c}0.311 \\
\pm \\
0.129\end{array}$ & & & & \\
\hline \multirow{6}{*}{$\mathrm{Cd}$} & I & $\begin{array}{c}0.018 \\
\pm \\
0.002 \\
\end{array}$ & $\begin{array}{c}0.021 \\
\pm \\
0.018 \\
\end{array}$ & $\begin{array}{c}0.025 \\
\pm \\
0.002 \\
\end{array}$ & $\begin{array}{c}0.014 \\
\pm \\
0.025 \\
\end{array}$ & $\begin{array}{c}0.020 \\
\pm \\
0.004 \\
\end{array}$ & & & & \\
\hline & II & N.D. & $\begin{array}{c}0.015 \\
\pm \\
0.031\end{array}$ & $\begin{array}{c}0.019 \\
\pm \\
0.007\end{array}$ & $\begin{array}{c}0.011 \\
\pm \\
0.02\end{array}$ & $\begin{array}{c}0.011 \\
\pm \\
0.007\end{array}$ & Site & 4 & 12.854 & 0 \\
\hline & III & $\begin{array}{c}0.009 \\
\pm \\
0.011\end{array}$ & $\begin{array}{c}0.018 \\
\pm \\
0.002\end{array}$ & $\begin{array}{c}0.022 \\
\pm \\
0.007\end{array}$ & $\begin{array}{c}0.014 \\
\pm \\
0.011\end{array}$ & $\begin{array}{c}0.016 \\
\pm \\
0.011 \\
\end{array}$ & Season & 3 & 4.607 & 0.007 \\
\hline & IV & $\begin{array}{c}0.016 \\
\pm \\
0.007\end{array}$ & $\begin{array}{c}0.026 \\
\pm \\
0.002\end{array}$ & $\begin{array}{c}0.031 \\
\pm \\
0.007\end{array}$ & $\begin{array}{c}0.021 \\
\pm \\
0.009\end{array}$ & $\begin{array}{c}0.024 \\
\pm \\
0.004\end{array}$ & Site $\mathrm{x}$ Season & 12 & 5.614 & 0 \\
\hline & V & $\begin{array}{c}0.021 \\
\pm \\
0.011\end{array}$ & $\begin{array}{c}0.031 \\
\pm \\
0.009\end{array}$ & $\begin{array}{c}0.038 \\
\pm \\
0.002\end{array}$ & $\begin{array}{c}0.027 \\
\pm \\
0.009\end{array}$ & $\begin{array}{c}0.029 \\
\pm \\
0.009\end{array}$ & & & & \\
\hline & Total & $\begin{array}{c}0.016 \\
\pm \\
0.008\end{array}$ & $\begin{array}{c}0.022 \\
\pm \\
0.012\end{array}$ & $\begin{array}{c}0.027 \\
\pm \\
0.005\end{array}$ & $\begin{array}{c}0.017 \\
\pm \\
0.015\end{array}$ & $\begin{array}{c}0.020 \\
\pm \\
0.007\end{array}$ & & & & \\
\hline \multirow{6}{*}{$\mathrm{Pb}$} & I & $\begin{array}{c}0.006 \\
\pm \\
0.002 \\
\end{array}$ & $\begin{array}{c}0.026 \\
\pm \\
0.011 \\
\end{array}$ & $\begin{array}{c}0.034 \\
\pm \\
0.002 \\
\end{array}$ & $\begin{array}{c}0.011 \\
\pm \\
0.002 \\
\end{array}$ & $\begin{array}{c}0.019 \\
\pm \\
0.007\end{array}$ & & & & \\
\hline & II & N.D. & $\begin{array}{c}0.011 \\
\pm \\
0.007\end{array}$ & $\begin{array}{c}0.017 \\
\pm \\
0.002\end{array}$ & $\begin{array}{c}0.006 \\
\pm \\
0.002\end{array}$ & $\begin{array}{c}0.009 \\
\pm \\
0.002\end{array}$ & Site & 4 & 11.707 & 0 \\
\hline & III & N.D. & $\begin{array}{c}0.017 \\
\pm \\
0.016\end{array}$ & $\begin{array}{c}0.029 \\
\pm \\
0.007 \\
\end{array}$ & $\begin{array}{c}0.008 \\
\pm \\
0.002\end{array}$ & $\begin{array}{c}0.014 \\
\pm \\
0.007\end{array}$ & Season & 3 & 4.601 & 0.007 \\
\hline & IV & $\begin{array}{c}0.008 \\
\pm \\
0.004\end{array}$ & $\begin{array}{c}0.032 \\
\pm \\
0.007\end{array}$ & $\begin{array}{c}0.054 \\
\pm \\
0.011\end{array}$ & $\begin{array}{c}0.020 \\
\pm \\
0.007\end{array}$ & $\begin{array}{c}0.029 \\
\pm \\
0.007\end{array}$ & Site $\mathrm{x}$ Season & 12 & 10.943 & 0 \\
\hline & V & $\begin{array}{c}0.012 \\
\pm \\
0.007\end{array}$ & $\begin{array}{c}0.046 \\
\pm \\
0.002\end{array}$ & $\begin{array}{c}0.074 \\
\pm \\
0.018\end{array}$ & $\begin{array}{c}0.029 \\
\pm \\
0.002\end{array}$ & $\begin{array}{c}0.040 \\
\pm \\
0.004\end{array}$ & & & & \\
\hline & Total & $\begin{array}{c}0.009 \\
\pm \\
0.004\end{array}$ & $\begin{array}{c}0.026 \\
\pm \\
0.009\end{array}$ & $\begin{array}{c}0.042 \\
\pm \\
0.008\end{array}$ & $\begin{array}{c}0.015 \\
\pm \\
0.003\end{array}$ & $\begin{array}{c}0.022 \\
\pm \\
0.005\end{array}$ & & & & \\
\hline
\end{tabular}

N.D. : not detected 
Table 2: Seasonal variations of heavy metals concentrations ( $\mu \mathrm{g} / \mathrm{g}$ dry weight) in plankton from Lake Manzala.

\begin{tabular}{|c|c|c|c|c|c|c|c|c|c|c|}
\hline \multirow[b]{2}{*}{ Elements } & \multirow[b]{2}{*}{ Site } & \multicolumn{5}{|c|}{ Seasons } & \multicolumn{4}{|c|}{ ANOVA } \\
\hline & & Winter & Spring & Summer & Autumn & Total & Factor & df & $\begin{array}{c}F \\
\text { value }\end{array}$ & Sig. \\
\hline \multirow{6}{*}{$\mathrm{Cu}$} & I & $\begin{array}{c}88.430 \\
\pm \\
13.830\end{array}$ & $\begin{array}{c}111.430 \\
\pm \\
25.160\end{array}$ & $\begin{array}{c}118.860 \\
\pm \\
23.050\end{array}$ & $\begin{array}{c}96.340 \\
\pm \\
15.370\end{array}$ & $\begin{array}{c}103.760 \\
\pm \\
18.180\end{array}$ & & & & \\
\hline & II & $\begin{array}{c}48.570 \\
\pm \\
21.230\end{array}$ & $\begin{array}{c}69.440 \\
\pm \\
8.780\end{array}$ & $\begin{array}{c}75.480 \\
\pm \\
31.840\end{array}$ & $\begin{array}{c}59.910 \\
\pm \\
20.670\end{array}$ & $\begin{array}{c}63.350 \\
\pm \\
25.360\end{array}$ & Site & 4 & 2.315 & 0.74 \\
\hline & III & $\begin{array}{c}71.890 \\
\pm \\
8.100\end{array}$ & $\begin{array}{c}84.860 \\
\pm \\
23.050\end{array}$ & $\begin{array}{c}93.830 \\
\pm \\
23.500\end{array}$ & $\begin{array}{c}78.000 \\
\pm \\
15.370\end{array}$ & $\begin{array}{c}82.140 \\
\pm \\
20.050\end{array}$ & Season & 3 & 1.142 & 0.344 \\
\hline & IV & $\begin{array}{c}90.570 \\
\pm \\
23.060\end{array}$ & $\begin{array}{c}115.750 \\
\pm \\
20.490\end{array}$ & $\begin{array}{c}135.720 \\
\pm \\
30.460\end{array}$ & $\begin{array}{c}104.000 \\
\pm \\
30.740\end{array}$ & $\begin{array}{c}111.510 \\
\pm \\
17.200\end{array}$ & $\begin{array}{l}\text { Site } x \\
\text { Season }\end{array}$ & 12 & 2.166 & 0.034 \\
\hline & V & $\begin{array}{c}108.760 \\
\pm \\
34.890 \\
\end{array}$ & $\begin{array}{c}136.620 \\
\pm \\
39.110 \\
\end{array}$ & $\begin{array}{c}154.430 \\
\pm \\
41.510\end{array}$ & $\begin{array}{c}126.000 \\
\pm \\
32.120\end{array}$ & $\begin{array}{c}131.450 \\
\pm \\
49.640\end{array}$ & & & & \\
\hline & Total & $\begin{array}{c}81.644 \\
\pm \\
20.222 \\
\end{array}$ & $\begin{array}{c}103.620 \\
\pm \\
23.318 \\
\end{array}$ & $\begin{array}{c}115.664 \\
\pm \\
30.072 \\
\end{array}$ & $\begin{array}{c}92.850 \\
\pm \\
22.854 \\
\end{array}$ & $\begin{array}{c}98.442 \\
\pm \\
26.086 \\
\end{array}$ & & & & \\
\hline \multirow{6}{*}{ Zn } & I & $\begin{array}{c}406.420 \\
\pm \\
140.220 \\
\end{array}$ & $\begin{array}{c}537.720 \\
\pm \\
71.710 \\
\end{array}$ & $\begin{array}{c}549.090 \\
\pm \\
97.020 \\
\end{array}$ & $\begin{array}{c}462.180 \\
\pm \\
89.950 \\
\end{array}$ & $\begin{array}{c}488.850 \\
\pm \\
168.740 \\
\end{array}$ & & & & \\
\hline & II & $\begin{array}{c}248.540 \\
\pm \\
126.800 \\
\end{array}$ & $\begin{array}{c}358.040 \\
\pm \\
64.740 \\
\end{array}$ & $\begin{array}{c}380.150 \\
\pm \\
92.860 \\
\end{array}$ & $\begin{array}{c}267.190 \\
\pm \\
185.200 \\
\end{array}$ & $\begin{array}{c}313.480 \\
\pm \\
115.400 \\
\end{array}$ & Site & 4 & 1.557 & 0.204 \\
\hline & III & $\begin{array}{c}251.640 \\
\pm \\
104.860\end{array}$ & $\begin{array}{c}428.960 \\
\pm \\
177.860\end{array}$ & $\begin{array}{c}460.650 \\
\pm \\
125.260\end{array}$ & $\begin{array}{c}390.410 \\
\pm \\
52.360\end{array}$ & $\begin{array}{c}382.910 \\
\pm \\
124.150\end{array}$ & Season & 3 & 2.386 & 0.083 \\
\hline & IV & $\begin{array}{c}430.670 \\
\pm \\
74.050\end{array}$ & $\begin{array}{c}610.280 \\
\pm \\
174.540\end{array}$ & $\begin{array}{c}695.730 \\
\pm \\
216.230\end{array}$ & $\begin{array}{c}520.140 \\
\pm \\
110.890\end{array}$ & $\begin{array}{c}564.210 \\
\pm \\
77.180\end{array}$ & $\begin{array}{l}\text { Site } x \\
\text { Season }\end{array}$ & 12 & 2.048 & 0.045 \\
\hline & V & $\begin{array}{c}490.850 \\
\pm \\
66.650\end{array}$ & $\begin{array}{c}620.280 \\
\pm \\
144.180\end{array}$ & $\begin{array}{c}737.650 \\
\pm \\
91.970\end{array}$ & $\begin{array}{c}560.460 \\
\pm \\
48.940\end{array}$ & $\begin{array}{c}602.310 \\
\pm \\
91.080\end{array}$ & & & & \\
\hline & Total & $\begin{array}{c}365.624 \\
\pm \\
102.516 \\
\end{array}$ & $\begin{array}{c}511.056 \\
\pm \\
126.606 \\
\end{array}$ & $\begin{array}{c}564.654 \\
\pm \\
124.668\end{array}$ & $\begin{array}{c}440.076 \\
\pm \\
97.468 \\
\end{array}$ & $\begin{array}{c}470.352 \\
\pm \\
115.310 \\
\end{array}$ & & & & \\
\hline \multirow{6}{*}{ Cd } & I & $\begin{array}{c}20.170 \\
\pm \\
1.620\end{array}$ & $\begin{array}{c}27.000 \\
\pm \\
6.450\end{array}$ & $\begin{array}{c}32.000 \\
\pm \\
8.940\end{array}$ & $\begin{array}{c}25.670 \\
\pm \\
8.600\end{array}$ & $\begin{array}{c}26.210 \\
\pm \\
9.400\end{array}$ & & & & \\
\hline & II & $\begin{array}{c}14.290 \\
\pm \\
7.750\end{array}$ & $\begin{array}{c}21.280 \\
\pm \\
2.450\end{array}$ & $\begin{array}{c}25.340 \\
\pm \\
7.750\end{array}$ & $\begin{array}{c}17.760 \\
\pm \\
3.270\end{array}$ & $\begin{array}{c}19.670 \\
\pm \\
9.970\end{array}$ & Site & 4 & 2.065 & 0.104 \\
\hline & III & $\begin{array}{c}18.610 \\
\pm \\
5.090\end{array}$ & $\begin{array}{c}26.000 \\
\pm \\
6.450\end{array}$ & $\begin{array}{c}30.430 \\
\pm \\
6.580\end{array}$ & $\begin{array}{c}22.670 \\
\pm \\
9.660\end{array}$ & $\begin{array}{c}24.430 \\
\pm \\
9.540\end{array}$ & Season & 3 & 2.350 & 0.087 \\
\hline & IV & $\begin{array}{c}24.820 \\
\pm \\
12.910\end{array}$ & $\begin{array}{c}33.220 \\
\pm \\
2.860\end{array}$ & $\begin{array}{c}36.670 \\
\pm \\
8.600\end{array}$ & $\begin{array}{c}29.470 \\
\pm \\
8.940\end{array}$ & $\begin{array}{c}31.040 \\
\pm \\
11.220\end{array}$ & $\begin{array}{c}\text { Site } x \\
\text { Season }\end{array}$ & 12 & 2.947 & 0.005 \\
\hline & V & $\begin{array}{c}27.380 \\
\pm \\
7.920\end{array}$ & $\begin{array}{c}39.970 \\
\pm \\
6.000\end{array}$ & $\begin{array}{c}46.000 \\
\pm \\
12.910\end{array}$ & $\begin{array}{c}31.270 \\
\pm \\
8.600\end{array}$ & $\begin{array}{c}36.150 \\
\pm \\
9.800\end{array}$ & & & & \\
\hline & Total & $\begin{array}{c}21.054 \\
\pm \\
7.058\end{array}$ & $\begin{array}{c}29.494 \\
\pm \\
4.842\end{array}$ & $\begin{array}{c}34.088 \\
\pm \\
8.956\end{array}$ & $\begin{array}{c}25.368 \\
\pm \\
7.814\end{array}$ & $\begin{array}{c}27.500 \\
\pm \\
9.986\end{array}$ & & & & \\
\hline \multirow{6}{*}{$\mathbf{P b}$} & I & $\begin{array}{c}56.750 \\
\pm \\
13.450\end{array}$ & $\begin{array}{c}79.130 \\
\pm \\
33.620\end{array}$ & $\begin{array}{c}91.130 \\
\pm \\
33.620\end{array}$ & $\begin{array}{c}69.530 \\
\pm \\
22.410\end{array}$ & $\begin{array}{c}74.130 \\
\pm \\
16.630\end{array}$ & & & & \\
\hline & II & $\begin{array}{c}44.750 \\
\pm \\
13.450\end{array}$ & $\begin{array}{c}61.210 \\
\pm \\
28.870 \\
\end{array}$ & $\begin{array}{c}72.050 \\
\pm \\
15.930\end{array}$ & $\begin{array}{c}55.310 \\
\pm \\
18.280\end{array}$ & $\begin{array}{c}58.330 \\
\pm \\
47.010 \\
\end{array}$ & Site & 4 & 3.644 & 0.013 \\
\hline & III & $\begin{array}{c}51.300 \\
\pm \\
2.040\end{array}$ & $\begin{array}{c}74.780 \\
\pm \\
27.500\end{array}$ & $\begin{array}{c}83.720 \\
\pm \\
22.460\end{array}$ & $\begin{array}{c}67.450 \\
\pm \\
24.820\end{array}$ & $\begin{array}{c}69.310 \\
\pm \\
24.510\end{array}$ & Season & 3 & 0.961 & 0.421 \\
\hline & IV & $\begin{array}{c}77.130 \\
\pm \\
33.620\end{array}$ & $\begin{array}{c}109.300 \\
\pm \\
34.350\end{array}$ & $\begin{array}{c}118.450 \\
\pm \\
51.640\end{array}$ & $\begin{array}{c}91.170 \\
\pm \\
44.830\end{array}$ & $\begin{array}{c}99.010 \\
\pm \\
27.270\end{array}$ & $\begin{array}{c}\text { Site } x \\
\text { Season }\end{array}$ & 12 & 0.628 & 0.806 \\
\hline & V & $\begin{array}{c}95.690 \\
\pm \\
25.180\end{array}$ & $\begin{array}{c}132.830 \\
\pm \\
43.050\end{array}$ & $\begin{array}{c}149.130 \\
\pm \\
33.620\end{array}$ & $\begin{array}{c}118.530 \\
\pm \\
53.930\end{array}$ & $\begin{array}{c}124.040 \\
\pm \\
31.460\end{array}$ & & & & \\
\hline & Total & $\begin{array}{c}65.124 \\
\pm \\
17.548\end{array}$ & $\begin{array}{c}91.450 \\
\pm \\
33.478\end{array}$ & $\begin{array}{c}102.896 \\
\pm \\
31.454\end{array}$ & $\begin{array}{c}80.398 \\
\pm \\
32.854\end{array}$ & $\begin{array}{c}84.964 \\
\pm \\
29.376\end{array}$ & & & & \\
\hline
\end{tabular}


Table.3: Seasonal variations of copper concentration $((\mu \mathrm{g} / \mathrm{g}$ dry weight $)$ in different organs of Liza aurata from Lake Manzala.

\begin{tabular}{|c|c|c|c|c|c|c|c|c|c|c|}
\hline \multirow{2}{*}{ Organ } & \multirow{2}{*}{ Site } & \multicolumn{5}{|c|}{ Seasons } & \multicolumn{4}{|c|}{ ANOVA } \\
\hline & & Winter & Spring & Summer & Autumn & Total & Factor & $\mathrm{df}$ & $\mathrm{F}$ value & Sig. \\
\hline \multirow{5}{*}{ Gills } & I & $\begin{array}{c}11.520 \\
\pm \\
0.610\end{array}$ & $\begin{array}{c}12.340 \\
\pm \\
0.770\end{array}$ & $\begin{array}{c}13.850 \\
\pm \\
1.250\end{array}$ & $\begin{array}{c}13.620 \\
\pm \\
0.280\end{array}$ & $\begin{array}{c}12.830 \\
\pm \\
1.230 \\
\end{array}$ & Site & 4 & 80.467 & 0 \\
\hline & II & $\begin{array}{c}10.510 \\
\pm \\
1.010\end{array}$ & $\begin{array}{c}11.660 \\
\pm \\
1.180\end{array}$ & $\begin{array}{c}12.770 \\
\pm \\
0.800\end{array}$ & $\begin{array}{c}12.580 \\
\pm \\
0.540\end{array}$ & $\begin{array}{c}11.880 \\
\pm \\
1.240\end{array}$ & Season & 3 & 48.159 & 0 \\
\hline & III & $\begin{array}{c}11.820 \\
\pm \\
0.580\end{array}$ & $\begin{array}{c}12.820 \\
\pm \\
1.030\end{array}$ & $\begin{array}{c}13.250 \\
\pm \\
0.700\end{array}$ & $\begin{array}{c}12.660 \\
\pm \\
0.840\end{array}$ & $\begin{array}{c}12.640 \\
\pm \\
0.910\end{array}$ & Organ & 2 & 3565.515 & 0 \\
\hline & IV & $\begin{array}{c}11.830 \\
\pm \\
0.810\end{array}$ & $\begin{array}{c}14.370 \\
\pm \\
1.810\end{array}$ & $\begin{array}{c}14.420 \\
\pm \\
2.890\end{array}$ & $\begin{array}{c}13.220 \\
\pm \\
0.670\end{array}$ & $\begin{array}{c}13.460 \\
\pm \\
1.970\end{array}$ & $\begin{array}{c}\text { Site } \mathrm{x} \\
\text { Season }\end{array}$ & 12 & 1.325 & 0.204 \\
\hline & V & $\begin{array}{c}14.220 \\
\pm \\
0.830\end{array}$ & $\begin{array}{c}16.510 \\
\pm \\
1.290\end{array}$ & $\begin{array}{c}16.520 \\
\pm \\
2.320\end{array}$ & $\begin{array}{c}14.680 \\
\pm \\
1.440\end{array}$ & $\begin{array}{c}15.480 \\
\pm \\
1.790\end{array}$ & $\begin{array}{l}\text { Site } \mathrm{x} \\
\text { Organ }\end{array}$ & 8 & 7.986 & 0 \\
\hline \multirow{5}{*}{ Skin } & I & $\begin{array}{c}7.780 \\
\pm \\
0.080\end{array}$ & $\begin{array}{c}7.870 \\
\pm \\
0.150\end{array}$ & $\begin{array}{c}8.940 \\
\pm \\
0.310\end{array}$ & $\begin{array}{c}8.870 \\
\pm \\
0.190\end{array}$ & $\begin{array}{c}8.370 \\
\quad \pm \\
0.590\end{array}$ & $\begin{array}{c}\text { Season x } \\
\text { Organ }\end{array}$ & 6 & 4.024 & 0.001 \\
\hline & II & $\begin{array}{c}6.730 \\
\pm \\
0.080 \\
\end{array}$ & $\begin{array}{c}6.820 \\
\pm \\
0.200\end{array}$ & $\begin{array}{c}7.930 \\
\pm \\
0.420\end{array}$ & $\begin{array}{c}7.340 \\
\pm \\
0.120\end{array}$ & $\begin{array}{c}7.200 \\
\quad \pm \\
0.540\end{array}$ & & & & \\
\hline & III & $\begin{array}{c}6.740 \\
\pm \\
0.570\end{array}$ & $\begin{array}{c}7.860 \\
\pm \\
0.230\end{array}$ & $\begin{array}{c}8.920 \\
\pm \\
0.530\end{array}$ & $\begin{array}{c}7.840 \\
\quad \pm \\
0.540\end{array}$ & $\begin{array}{c}7.840 \\
\pm \\
0.910\end{array}$ & Site & & & \\
\hline & IV & $\begin{array}{c}7.830 \\
\pm \\
0.150\end{array}$ & $\begin{array}{c}8.920 \\
\pm \\
0.200\end{array}$ & $\begin{array}{c}8.960 \\
\pm \\
0.290\end{array}$ & $\begin{array}{c}8.860 \\
\pm \\
\pm .070\end{array}$ & $\begin{array}{c}8.640 \\
\pm \\
0.510\end{array}$ & $\mathrm{x}$ & & & \\
\hline & $\mathrm{V}$ & $\begin{array}{c}8.960 \\
\pm \\
0.210 \\
\end{array}$ & $\begin{array}{c}9.210 \\
\pm \\
0.280 \\
\end{array}$ & $\begin{array}{c}9.710 \\
\pm \\
0.490 \\
\end{array}$ & $\begin{array}{c}9.590 \\
\pm \\
0.450\end{array}$ & $\begin{array}{c}9.370 \\
\pm \\
0.460\end{array}$ & Season & & & \\
\hline \multirow{5}{*}{ Muscles } & I & $\begin{array}{c}3.430 \\
\pm \\
0.250\end{array}$ & $\begin{array}{c}3.960 \\
\pm \\
0.240\end{array}$ & $\begin{array}{c}4.700 \\
\pm \\
0.230\end{array}$ & $\begin{array}{c}3.880 \\
\pm \\
0.220\end{array}$ & $\begin{array}{c}3.990 \\
\pm \\
0.510\end{array}$ & $\mathrm{x}$ & & & \\
\hline & II & $\begin{array}{c}3.610 \\
\pm \\
0.480\end{array}$ & $\begin{array}{c}3.810 \\
\pm \\
0.110\end{array}$ & $\begin{array}{c}4.030 \\
\pm \\
0.260\end{array}$ & $\begin{array}{c}3.550 \\
\pm \\
0.140\end{array}$ & $\begin{array}{c}3.750 \\
\pm \\
0.330\end{array}$ & Organ & 24 & 1.555 & 0.052 \\
\hline & III & $\begin{array}{c}3.460 \\
\pm \\
0.210\end{array}$ & $\begin{array}{c}3.990 \\
\pm \\
0.340\end{array}$ & $\begin{array}{c}4.150 \\
\pm \\
0.190\end{array}$ & $\begin{array}{c}4.110 \\
\pm \\
0.210\end{array}$ & $\begin{array}{c}3.930 \\
\pm \\
0.360\end{array}$ & & & & \\
\hline & IV & $\begin{array}{c}3.990 \\
\pm \\
0.300\end{array}$ & $\begin{array}{c}4.570 \\
\pm \\
0.120\end{array}$ & $\begin{array}{c}4.740 \\
\pm \\
0.410\end{array}$ & $\begin{array}{c}4.520 \\
\pm \\
0.180\end{array}$ & $\begin{array}{c}4.460 \\
\pm \\
0.380\end{array}$ & & & & \\
\hline & $\mathrm{V}$ & $\begin{array}{c}4.000 \\
\pm \\
0.270\end{array}$ & $\begin{array}{c}4.930 \\
\pm \\
0.320\end{array}$ & $\begin{array}{c}5.490 \\
\pm \\
0.150\end{array}$ & $\begin{array}{c}5.430 \\
\pm \\
0.280\end{array}$ & $\begin{array}{c}4.960 \\
\pm \\
0.660\end{array}$ & & & & \\
\hline
\end{tabular}


Table 4: Seasonal variations of Zinc concentration ( $\mu \mathrm{g} / \mathrm{g}$ dry weight) in different organs of Liza aurata from Lake Manzala.

\begin{tabular}{|c|c|c|c|c|c|c|c|c|c|c|}
\hline \multirow{2}{*}{ Organ } & \multirow{2}{*}{ Site } & \multicolumn{5}{|c|}{ Seasons } & \multicolumn{4}{|c|}{ ANOVA } \\
\hline & & Winter & Spring & Summer & Autumn & Total & Factor & $\mathrm{df}$ & F value & Sig. \\
\hline \multirow{5}{*}{ Gills } & I & $\begin{array}{c}52.720 \\
\pm \\
5.620\end{array}$ & $\begin{array}{c}75.360 \\
\pm \\
2.970\end{array}$ & $\begin{array}{c}86.250 \\
\pm \\
2.980\end{array}$ & $\begin{array}{c}68.460 \\
\pm \\
4.100\end{array}$ & $\begin{array}{c}70.700 \\
\pm \\
13.030\end{array}$ & Site & 4 & 748.475 & 0 \\
\hline & II & $\begin{array}{c}47.410 \\
\pm \\
2.910\end{array}$ & $\begin{array}{c}66.740 \\
\pm \\
3.490\end{array}$ & $\begin{array}{c}74.660 \\
\pm \\
2.710\end{array}$ & $\begin{array}{c}59.260 \\
\pm \\
3.170\end{array}$ & $\begin{array}{c}62.020 \\
\pm \\
10.680\end{array}$ & Season & 3 & 1091.732 & 0 \\
\hline & III & $\begin{array}{c}52.640 \\
\pm \\
3.110\end{array}$ & $\begin{array}{c}74.450 \\
\pm \\
2.940\end{array}$ & $\begin{array}{c}81.730 \\
\pm \\
3.200\end{array}$ & $\begin{array}{c}67.290 \\
\pm \\
4.460\end{array}$ & $\begin{array}{c}69.030 \\
\pm \\
11.480\end{array}$ & Organ & 2 & 9358.979 & 0 \\
\hline & IV & $\begin{array}{c}64.350 \\
\pm \\
3.530 \\
\end{array}$ & $\begin{array}{c}91.580 \\
\pm \\
2.410 \\
\end{array}$ & $\begin{array}{c}103.470 \\
\pm \\
2.470 \\
\end{array}$ & $\begin{array}{c}79.630 \\
\pm \\
2.370 \\
\end{array}$ & $\begin{array}{c}84.760 \\
\pm \\
15.070 \\
\end{array}$ & $\begin{array}{l}\text { Site } \mathrm{x} \\
\text { Season }\end{array}$ & 12 & 21.840 & 0 \\
\hline & V & $\begin{array}{c}67.210 \\
\pm \\
3.630\end{array}$ & $\begin{array}{c}101.360 \\
\pm \\
3.810\end{array}$ & $\begin{array}{c}136.170 \\
\pm \\
4.260 \\
\end{array}$ & $\begin{array}{c}94.460 \\
\pm \\
3.010 \\
\end{array}$ & $\begin{array}{c}99.800 \\
\pm \\
25.440\end{array}$ & $\begin{array}{l}\text { Site } x \\
\text { Organ }\end{array}$ & 8 & 53.910 & 0 \\
\hline \multirow{5}{*}{ Skin } & I & $\begin{array}{c}35.310 \\
\pm \\
3.820\end{array}$ & $\begin{array}{c}54.350 \\
\pm \\
2.490\end{array}$ & $\begin{array}{c}60.280 \\
\pm \\
2.510\end{array}$ & $\begin{array}{c}46.980 \\
\pm \\
1.030\end{array}$ & $\begin{array}{c}49.230 \\
\pm \\
9.860\end{array}$ & $\begin{array}{c}\text { Season } \mathrm{x} \\
\text { Organ }\end{array}$ & 6 & 132.850 & 0 \\
\hline & II & $\begin{array}{c}30.630 \\
\pm \\
3.700\end{array}$ & $\begin{array}{c}42.700 \\
\pm \\
2.430\end{array}$ & $\begin{array}{c}51.340 \\
\pm \\
2.590\end{array}$ & $\begin{array}{c}39.370 \\
\pm \\
2.460\end{array}$ & $\begin{array}{c}41.010 \\
\pm \\
8.040\end{array}$ & & & & \\
\hline & III & $\begin{array}{c}33.720 \\
\pm \\
2.250\end{array}$ & $\begin{array}{c}54.660 \\
\pm \\
2.920 \\
\end{array}$ & $\begin{array}{c}65.630 \\
\pm \\
3.230 \\
\end{array}$ & $\begin{array}{c}41.460 \\
\pm \\
2.780 \\
\end{array}$ & $\begin{array}{c}48.870 \\
\pm \\
12.820\end{array}$ & Site & & & \\
\hline & IV & $\begin{array}{c}41.420 \\
\pm \\
2.690 \\
\end{array}$ & $\begin{array}{c}62.560 \\
\pm \\
2.430 \\
\end{array}$ & $\begin{array}{c}78.240 \\
\pm \\
3.490 \\
\end{array}$ & $\begin{array}{c}52.260 \\
\pm \\
2.730 \\
\end{array}$ & $\begin{array}{c}58.620 \\
\pm \\
14.140 \\
\end{array}$ & $\mathrm{x}$ & & & \\
\hline & $\mathrm{V}$ & $\begin{array}{c}7.520 \\
\pm \\
2.530 \\
\end{array}$ & $\begin{array}{c}72.460 \\
\pm \\
2.810 \\
\end{array}$ & $\begin{array}{c}85.380 \\
\pm \\
3.170 \\
\end{array}$ & $\begin{array}{c}61.360 \\
\pm \\
3.620 \\
\end{array}$ & $\begin{array}{c}66.680 \\
\pm \\
14.590\end{array}$ & Season & & & \\
\hline \multirow{5}{*}{ Muscles } & I & $\begin{array}{c}15.500 \\
\pm \\
1.520\end{array}$ & $\begin{array}{c}19.660 \\
\pm \\
2.020\end{array}$ & $\begin{array}{c}24.060 \\
\pm \\
1.870\end{array}$ & $\begin{array}{c}18.340 \\
\pm \\
2.860\end{array}$ & $\begin{array}{c}19.390 \\
\pm \\
3.720\end{array}$ & $\mathrm{x}$ & & & \\
\hline & II & $\begin{array}{c}12.460 \\
\pm \\
1.610\end{array}$ & $\begin{array}{c}18.070 \\
\pm \\
1.480\end{array}$ & $\begin{array}{c}17.460 \\
\pm \\
1.490\end{array}$ & $\begin{array}{c}14.270 \\
\pm \\
1.860\end{array}$ & $\begin{array}{c}15.570 \\
\pm \\
2.790\end{array}$ & Organ & 24 & 9.000 & 0 \\
\hline & III & $\begin{array}{c}12.980 \\
\pm \\
2.360\end{array}$ & $\begin{array}{c}19.680 \\
\pm \\
2.250\end{array}$ & $\begin{array}{c}21.290 \\
\pm \\
2.540\end{array}$ & $\begin{array}{c}16.760 \\
\pm \\
2.080\end{array}$ & $\begin{array}{c}17.680 \\
\pm \\
3.880\end{array}$ & & & & \\
\hline & IV & $\begin{array}{c}19.410 \\
\pm \\
2.470 \\
\end{array}$ & $\begin{array}{c}27.760 \\
\pm \\
2.420 \\
\end{array}$ & $\begin{array}{c}29.780 \\
\pm \\
2.320 \\
\end{array}$ & $\begin{array}{c}22.440 \\
\pm \\
2.270 \\
\end{array}$ & $\begin{array}{c}24.850 \\
\pm \\
4.760 \\
\end{array}$ & & & & \\
\hline & V & $\begin{array}{c}25.380 \\
\pm \\
3.190\end{array}$ & $\begin{array}{c}30.240 \\
\pm \\
1.850\end{array}$ & $\begin{array}{c}35.450 \\
\pm \\
3.340\end{array}$ & $\begin{array}{c}27.640 \\
\pm \\
2.300\end{array}$ & $\begin{array}{c}29.680 \\
\pm \\
4.600\end{array}$ & & & & \\
\hline
\end{tabular}


Table 5: Seasonal variations of cadmium concentration ( $\mu \mathrm{g} / \mathrm{g}$ dry weight) in different organs of Liza aurata from Lake Manzala.

\begin{tabular}{|c|c|c|c|c|c|c|c|c|c|c|}
\hline \multirow{2}{*}{ Organ } & \multirow{2}{*}{ Site } & \multicolumn{5}{|c|}{ Seasons } & \multicolumn{4}{|c|}{ ANOVA } \\
\hline & & Winter & Spring & Summer & Autumn & Total & Factor & $\mathrm{df}$ & F value & Sig. \\
\hline \multirow{5}{*}{ Gills } & I & $\begin{array}{c}3.350 \\
\pm \\
0.590\end{array}$ & $\begin{array}{c}3.620 \\
\pm \\
0.820\end{array}$ & $\begin{array}{c}4.190 \\
\pm \\
0.470 \\
\end{array}$ & $\begin{array}{c}2.920 \\
\pm \\
0.700\end{array}$ & $\begin{array}{c}3.520 \\
\pm \\
0.770 \\
\end{array}$ & Site & 4 & 67.547 & 0 \\
\hline & II & $\begin{array}{c}2.630 \\
\pm \\
0.520 \\
\end{array}$ & $\begin{array}{c}3.360 \\
\pm \\
0.550 \\
\end{array}$ & $\begin{array}{c}3.400 \\
\pm \\
0.310 \\
\end{array}$ & 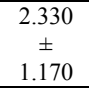 & $\begin{array}{c}2.930 \\
\pm \\
0.810 \\
\end{array}$ & Season & 3 & 34.024 & 0 \\
\hline & III & $\begin{array}{c}3.170 \\
\pm \\
0.370 \\
\end{array}$ & $\begin{array}{c}3.970 \\
\pm \\
0.450 \\
\end{array}$ & 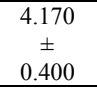 & $\begin{array}{c}3.290 \\
\pm \\
0.260 \\
\end{array}$ & $\begin{array}{c}3.650 \\
\pm \\
0.560 \\
\end{array}$ & Organ & 2 & 1152.758 & 0 \\
\hline & IV & $\begin{array}{c}4.180 \\
\pm \\
0.720 \\
\end{array}$ & $\begin{array}{c}.090 \\
\pm \\
0.830 \\
\end{array}$ & $\begin{array}{c}.420 \\
\pm \\
0.840 \\
\end{array}$ & 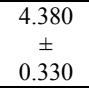 & $\begin{array}{c}4.770 \\
\pm \\
0.830\end{array}$ & $\begin{array}{c}\text { Site } \mathrm{x} \\
\text { Season }\end{array}$ & 12 & 0.691 & 0.759 \\
\hline & $\mathrm{V}$ & $\begin{array}{c}4.570 \\
\pm \\
0.740\end{array}$ & $\begin{array}{c}5.280 \\
\pm \\
0.620\end{array}$ & $\begin{array}{c}6.060 \\
\pm \\
1.160 \\
\end{array}$ & $\begin{array}{c}\begin{array}{c}4.850 \\
\pm \\
1.230\end{array} \\
\end{array}$ & $\begin{array}{c}5.190 \\
\pm \\
1.060\end{array}$ & $\begin{array}{l}\text { Site } \mathrm{x} \\
\text { Organ }\end{array}$ & 8 & 19.836 & 0 \\
\hline \multirow{5}{*}{ Skin } & I & $\begin{array}{c}1.620 \\
\pm \\
0.120 \\
\end{array}$ & $\begin{array}{c}2.140 \\
\pm \\
0.090 \\
\end{array}$ & $\begin{array}{c}2.210 \\
\pm \\
0.110 \\
\end{array}$ & $\begin{array}{c}1.770 \\
\pm \\
0.160 \\
\end{array}$ & $\begin{array}{c}1.940 \\
\pm \\
0.280 \\
\end{array}$ & $\begin{array}{c}\text { Season } \mathrm{x} \\
\text { Organ }\end{array}$ & 6 & 7.364 & 0 \\
\hline & II & $\begin{array}{c}1.590 \\
\pm \\
0.070 \\
\end{array}$ & $\begin{array}{c}1.730 \\
\pm \\
0.090 \\
\end{array}$ & $\begin{array}{c}1.720 \\
\pm \\
0.090 \\
\end{array}$ & $\begin{array}{c}1.640 \\
\pm \\
0.200 \\
\end{array}$ & $\begin{array}{c}1.670 \\
\pm \\
0.130\end{array}$ & & & & \\
\hline & III & $\begin{array}{c}1.520 \\
\pm \\
0.070 \\
\end{array}$ & $\begin{array}{c}2.170 \\
\pm \\
0.090\end{array}$ & $\begin{array}{c}1.870 \\
\pm \\
0.150 \\
\end{array}$ & $\begin{array}{c}1.680 \\
\pm \\
0.140\end{array}$ & $\begin{array}{c}1.810 \\
\pm \\
0.270\end{array}$ & Site & & & \\
\hline & IV & $\begin{array}{c}1.670 \\
\pm \\
0.090 \\
\end{array}$ & $\begin{array}{c}2.280 \\
\pm \\
0.320 \\
\end{array}$ & $\begin{array}{c}2.340 \\
\pm \\
0.090 \\
\end{array}$ & $\begin{array}{c}1.970 \\
\pm \\
0.130 \\
\end{array}$ & $\begin{array}{c}2.070 \\
\pm \\
0.320 \\
\end{array}$ & $\mathrm{x}$ & & & \\
\hline & $\mathrm{V}$ & $\begin{array}{c}2.070 \\
\pm \\
0.410 \\
\end{array}$ & $\begin{array}{c}2.560 \\
\pm \\
0.270\end{array}$ & $\begin{array}{c}2.760 \\
\pm \\
0.130 \\
\end{array}$ & $\begin{array}{c}2.520 \\
\pm \\
0.270 \\
\end{array}$ & $\begin{array}{c}2.480 \\
\pm \\
0.370\end{array}$ & Season & & & \\
\hline \multirow{5}{*}{ Muscles } & I & $\begin{array}{c}0.970 \\
\pm \\
0.370 \\
\end{array}$ & $\begin{array}{c}1.060 \\
\pm \\
0.210\end{array}$ & $\begin{array}{c}1.390 \\
\pm \\
0.150\end{array}$ & $\begin{array}{c}1.110 \\
\pm \\
0.070\end{array}$ & $\begin{array}{c}1.130 \\
\pm \\
0.270\end{array}$ & $\mathrm{x}$ & & & \\
\hline & II & $\begin{array}{c}0.810 \\
\pm \\
0.120\end{array}$ & $\begin{array}{c}0.970 \\
\pm \\
0.060\end{array}$ & $\begin{array}{c}1.140 \\
\pm \\
0.080\end{array}$ & $\begin{array}{c}1.070 \\
\pm \\
0.100\end{array}$ & $\begin{array}{c}1.000 \\
\pm \\
0.150\end{array}$ & Organ & 24 & 0.411 & 0.994 \\
\hline & III & $\begin{array}{c}0.960 \\
\pm \\
0.120\end{array}$ & $\begin{array}{c}1.130 \\
\pm \\
0.060\end{array}$ & $\begin{array}{c}1.230 \\
\pm \\
0.070\end{array}$ & $\begin{array}{c}1.110 \\
\pm \\
0.070\end{array}$ & $\begin{array}{c}1.110 \\
\pm \\
0.130\end{array}$ & & & & \\
\hline & IV & $\begin{array}{c}1.190 \\
\pm \\
0.150\end{array}$ & $\begin{array}{c}1.260 \\
\pm \\
0.060\end{array}$ & $\begin{array}{c}1.420 \\
\pm \\
0.090\end{array}$ & $\begin{array}{c}1.170 \\
\pm \\
0.100\end{array}$ & $\begin{array}{c}1.260 \\
\pm \\
0.140\end{array}$ & & & & \\
\hline & $\mathrm{V}$ & $\begin{array}{c}1.270 \\
\pm \\
0.090\end{array}$ & $\begin{array}{c}1.340 \\
\pm \\
0.060\end{array}$ & $\begin{array}{c}1.610 \\
\pm \\
0.160\end{array}$ & $\begin{array}{c}1.230 \\
\pm \\
0.090\end{array}$ & $\begin{array}{c}1.370 \\
\pm \\
0.180\end{array}$ & & & & \\
\hline
\end{tabular}


Table 6: Seasonal variations of lead concentration ( $\mu \mathrm{g} / \mathrm{g}$ dry weight) in different organs of Liza aurata from Lake Manzala.

\begin{tabular}{|c|c|c|c|c|c|c|c|c|c|c|}
\hline \multirow{2}{*}{ Organ } & \multirow{2}{*}{ Site } & \multicolumn{5}{|c|}{ Seasons } & \multicolumn{4}{|c|}{ ANOVA } \\
\hline & & Winter & Spring & Summer & Autumn & Total & Factor & $\mathrm{df}$ & F value & Sig. \\
\hline \multirow{5}{*}{ Gills } & I & $\begin{array}{c}7.140 \\
\pm \\
0.760\end{array}$ & $\begin{array}{c}8.720 \\
\pm \\
0.420\end{array}$ & $\begin{array}{c}9.320 \\
\pm \\
0.680\end{array}$ & $\begin{array}{c}8.480 \\
\pm \\
0.590\end{array}$ & $\begin{array}{c}8.420 \\
\pm \\
1.000\end{array}$ & Site & 4 & 104.379 & 0 \\
\hline & II & $\begin{array}{c}6.440 \\
\pm \\
0.670\end{array}$ & $\begin{array}{c}7.020 \\
\pm \\
0.490\end{array}$ & $\begin{array}{c}7.660 \\
\pm \\
0.560\end{array}$ & $\begin{array}{c}6.480 \\
\pm \\
0.570\end{array}$ & $\begin{array}{c}6.900 \\
\pm \\
0.730\end{array}$ & Season & 3 & 40.010 & 0 \\
\hline & III & $\begin{array}{c}6.940 \\
\pm \\
0.470\end{array}$ & $\begin{array}{c}7.880 \\
\pm \\
0.540\end{array}$ & $\begin{array}{c}8.320 \\
\pm \\
0.740\end{array}$ & $\begin{array}{c}7.360 \\
\pm \\
0.630\end{array}$ & $\begin{array}{c}7.630 \\
\pm \\
0.770\end{array}$ & Organ & 2 & 5872.010 & 0 \\
\hline & IV & $\begin{array}{c}8.120 \\
\pm \\
0.700\end{array}$ & $\begin{array}{c}9.460 \\
\pm \\
0.650\end{array}$ & $\begin{array}{c}9.840 \\
\pm \\
0.670\end{array}$ & $\begin{array}{c}9.080 \\
\pm \\
0.740\end{array}$ & $\begin{array}{c}9.130 \\
\pm \\
0.910\end{array}$ & $\begin{array}{l}\text { Site } \mathrm{x} \\
\text { Season }\end{array}$ & 12 & 0.504 & 0.911 \\
\hline & V & $\begin{array}{c}9.460 \\
\pm \\
0.680\end{array}$ & $\begin{array}{c}10.280 \\
\pm \\
0.350\end{array}$ & $\begin{array}{c}10.940 \\
\pm \\
0.670\end{array}$ & $\begin{array}{c}10.380 \\
\pm \\
0.580\end{array}$ & $\begin{array}{c}10.270 \\
\pm \\
0.760\end{array}$ & $\begin{array}{l}\text { Site } \mathrm{x} \\
\text { Organ }\end{array}$ & 8 & 34.132 & 0 \\
\hline \multirow{5}{*}{ Skin } & I & $\begin{array}{c}2.440 \\
\pm \\
0.310\end{array}$ & $\begin{array}{c}2.780 \\
\pm \\
0.380 \\
\end{array}$ & $\begin{array}{c}2.950 \\
\pm \\
0.310\end{array}$ & $\begin{array}{c}2.620 \\
\pm \\
0.620\end{array}$ & $\begin{array}{c}2.700 \\
\pm \\
0.440\end{array}$ & $\begin{array}{c}\text { Season } \mathrm{x} \\
\text { Organ }\end{array}$ & 6 & 9.482 & 0 \\
\hline & II & $\begin{array}{c}2.340 \\
\pm \\
0.240\end{array}$ & $\begin{array}{c}2.490 \\
\pm \\
0.530\end{array}$ & $\begin{array}{c}2.630 \\
\pm \\
0.370\end{array}$ & $\begin{array}{c}2.410 \\
\pm \\
0.380\end{array}$ & $\begin{array}{c}2.470 \\
\pm \\
0.370\end{array}$ & & & & \\
\hline & III & $\begin{array}{c}2.420 \\
\pm \\
0.340\end{array}$ & $\begin{array}{c}2.610 \\
\pm \\
0.360\end{array}$ & $\begin{array}{c}2.860 \\
\pm \\
0.320\end{array}$ & $\begin{array}{c}2.530 \\
\pm \\
0.350\end{array}$ & $\begin{array}{c}2.610 \\
\pm \\
0.350\end{array}$ & Site & & & \\
\hline & IV & $\begin{array}{c}2.620 \\
\pm \\
0.360\end{array}$ & $\begin{array}{c}2.860 \\
\pm \\
0.350\end{array}$ & $\begin{array}{c}3.070 \\
\pm \\
0.310\end{array}$ & $\begin{array}{c}2.720 \\
\pm \\
0.430\end{array}$ & $\begin{array}{c}2.820 \\
\pm \\
0.380\end{array}$ & $\mathrm{x}$ & & & \\
\hline & V & $\begin{array}{c}2.750 \\
\pm \\
0.310\end{array}$ & $\begin{array}{c}3.070 \\
\pm \\
0.430\end{array}$ & $\begin{array}{c}3.270 \\
\pm \\
0.380\end{array}$ & $\begin{array}{c}2.810 \\
\pm \\
0.370\end{array}$ & $\begin{array}{c}2.980 \\
\pm \\
0.410\end{array}$ & Season & & & \\
\hline \multirow{5}{*}{ Muscles } & I & $\begin{array}{c}1.720 \\
\pm \\
0.370\end{array}$ & $\begin{array}{c}1.900 \\
\pm \\
0.390\end{array}$ & $\begin{array}{c}2.110 \\
\pm \\
0.300\end{array}$ & $\begin{array}{c}1.920 \\
\pm \\
0.220\end{array}$ & $\begin{array}{c}1.910 \\
\pm \\
0.330\end{array}$ & $\mathrm{x}$ & & & \\
\hline & II & $\begin{array}{c}1.410 \\
\pm \\
0.200\end{array}$ & $\begin{array}{c}1.620 \\
\pm \\
0.520\end{array}$ & $\begin{array}{c}1.750 \\
\pm \\
0.180\end{array}$ & $\begin{array}{c}1.540 \\
\pm \\
0.190\end{array}$ & $\begin{array}{c}1.580 \\
\pm \\
0.310\end{array}$ & Organ & 24 & 0.464 & 0.986 \\
\hline & III & $\begin{array}{c}1.540 \\
\pm \\
0.300\end{array}$ & $\begin{array}{c}1.820 \\
\pm \\
0.400\end{array}$ & $\begin{array}{c}1.940 \\
\pm \\
0.350\end{array}$ & $\begin{array}{c}1.750 \\
\pm \\
0.280\end{array}$ & $\begin{array}{c}1.760 \\
\pm \\
0.340\end{array}$ & & & & \\
\hline & IV & $\begin{array}{c}1.970 \\
\pm \\
0.480\end{array}$ & $\begin{array}{c}2.160 \\
\pm \\
0.360\end{array}$ & $\begin{array}{c}2.370 \\
\pm \\
0.370\end{array}$ & $\begin{array}{c}2.130 \\
\pm \\
0.320\end{array}$ & $\begin{array}{c}2.160 \\
\pm \\
0.380\end{array}$ & & & & \\
\hline & V & $\begin{array}{c}2.190 \\
\pm \\
0.460\end{array}$ & $\begin{array}{c}2.470 \\
\pm \\
0.230\end{array}$ & $\begin{array}{c}2.660 \\
\pm \\
0.360\end{array}$ & $\begin{array}{c}2.420 \\
\pm \\
0.430\end{array}$ & $\begin{array}{c}2.440 \\
\pm \\
0.390\end{array}$ & & & & \\
\hline
\end{tabular}

